\title{
COMPORTAMENTO DE LINHAGENS DE FEIJÃO-MUNGO (Vigna radiata L.) EM SANTA CATARINA
}

\author{
BEHAVIOUR OF MUNGBEAN (Vigna radiata L.) IN SANTA CATARINA
}

\author{
Shiow Shong Lin ${ }^{1}$ Antonio Carlos Alves ${ }^{2}$
}

RESUMO

Foram conduzidos três experimentos na fazenda experimental do Centro de Ciências Agrárias da Universidade Federal de Santa Catarina, Florianópolis, SC, Brasil. A finalidade do trabalho foi avaliar o comportamento de 29 linhagens de feijão mungo provenientes do "Asian Vegetable Research and Development Center" (AVRDC). Avaliaram-se as características agronômicas (formacão da primeira flor, primeiro legume maduro, estatura da planta), o peso de mil sementes e o rendimento. As linhagens foram testadas em blocos ao acaso, com quatro repetições. Cada parcela foi constituída por 4 linhas de $6 m$ de comprimento, espaçadas de $50 \mathrm{~cm}$, com vinte sementes por metro de sulco. A floração inicial das linhagens ocorreu entre os 33 e 49 dias após o plantio, enquanto a maturação do primeiro legume se deu entre os 70 e 78 dias após a semeadura. A estatura das plantas e o peso de mil sementes variaram entre 22,5 e 63,8cm e 33,0 e 76,4g respectivamente. As linhagens mais produtivas foram: VC $3890 B, V C 1973 C$ e VC $2764 B$ com rendimento acima de $1140 \mathrm{~kg} . \mathrm{ha}^{-1}$. Estas linhagens apresentaram peso de mil sementes e estatura de planta boas para cultivo e um ciclo biológico médio. A colheita foi feita em uma única operação, entre 80 e 85 dias após a semeadura no primeiro e segundo experimentos. No terceiro experimento, a colheita foi feita em duas vezes, aos 80 e 85 dias e aos 95 e 100 dias, sendo que na primeira colheita foram colhidos $78 \%$ dos legumes; os restantes 22\% foram colhidos 15 dias após a primeira.

Palavras-chave: características agronômicas, peso de mil sementes, rendimento

\section{SUMMARY}

Three experiments were conducted at the experimental farm of the Agricultural Science Center, Federal University of Santa Catarina, Ressacada, Florianópolis, SC.
Brazil. Twenty nine lines of mungbean from the Asian Vegetable Research and Development Center (AVRDC), Taiwan, Republic of China were evaluated about their agronomic characters (first flower formation, first legume mature, plant height), thousand seeds weight and yield. The experimental design was randomized complete blocks with four replications, in plots consisting of four rows spaced $0.5 \mathrm{~m}$ of each other, with 20 seeds $\mathrm{m}^{-1}$. The initial flowering of mungbean lines occurred from 33 to 49 days after seeding, while the first legume mature appeared from 70 to 78 days after seeding. The plant height varied from 22.5 to $63.8 \mathrm{~cm}$ and the thousand seeds weight from 33.0 to $76.4 \mathrm{~g}$. The more productive lines were VC $3890 B, V C 1973 C$ and VC $2764 B$ with their yields higher than $1140 \mathrm{~kg} \mathrm{ha}^{-1}$. These lines showed their moderate value in plant height and weight of thousand seeds and not very early in maturing. In the first and second experiment, the only one harvest was made from 80 to 85 days after seeding. In the third experiment, the harvest was made two times, $78 \%$ of total grain production was obtained in the first harvesting and the rest of $22 \%$ was obtained 15 days after the first harvesting.

Key words: agronomic characters, thousand seeds weight, yield.

\section{INTRODUÇÃO}

O feijão-mungo é uma leguminosa importante, plantada extensivamente como fonte de alimentos e para uso industrial nas regiões tropicais e subtropicais. Estima-se que 1,5 milhões de toneladas de feijão-mungo são produzidas anualmente no mundo, em aproximadamente 3,8 milhões de hectares, sendo a Índia o maior produtor mundial, seguida pela Tailândia (VIEIRA, 1992). A

\footnotetext{
${ }^{1}$ Engenheiro Agrônomo, PhD., Professor Titular do Centro de Ciências Agrárias (CCA), Universidade Federal de Santa Catarina (UFSC), CP 476, 88040-900, Florianópolis, SC. E-mail: shiolin@cca.ufsc.br. Autor para correspondência.

${ }^{2}$ Engenheiro Agrônomo, Doutor, Professor Adjunto do CCA, UFSC, CP 476, 88040-900, Florianópolis, SC. E-mail:
} alves@cca.ufsc.br. 
produção dessa espécie no Brasil é pequena, mas vem crescendo devido ao aumento da demanda de grãos para a produção de brotos de feijão "Moyashi" (BARRADAS et al., 1989).

A cultura do feijão-mungo apresenta características que evidenciam um potencial uso agronômico, destacando-se o fácil plantio, o ciclo curto e a estabilidade da rentabilidade (SANGAKKARA \& SOMARATNE, 1988). Após a última colheita, os restos culturais do feijão-mungo encontram-se verdes, com alto teor de nitrogênio, podendo ser incorporados ao solo, melhorando, assim, suas características físicas e químicas, principalmente onde esses apresentam problemas de degradação. Estas características tornam o feijãomungo uma cultura de especial importância para pequenos produtores.

Essa leguminosa apresenta grande valor protéico, podendo ser utilizado como fonte protéica suplementar para o arroz (TSOU \& HSU, 1978). Além da riqueza em proteínas, ele apresenta bons teores de vitamina B e ferro (DUQUE et al., 1987).

No Brasil, estudos sobre o
comportamento do feijão-mungo foram feitos em Itaquaí, RJ, (DUQUE et al., 1987) e na Zona da Mata de Minas Gerais (VIEIRA, 1989). No Rio de Janeiro, DUQUE et al. (1987), estudaram 21 cultivares no plantio da "seca" em março e verificaram que o rendimento de grãos secos variou de 1154 a $1796 \mathrm{~kg} \mathrm{ha}^{-1}$ e que, para a maioria das cultivares, duas colheitas entre 63 e 69 dias após o plantio, foram atingidos $85-90 \%$ de legumes maduros. Na Zona da Mata de Minas Gerais, VIEIRA (1989) verificou que a produtividade do feijão-mungo verde foi semelhante à do feijão comum no plantio das "águas" e superior a ele na "seca".

A introdução e a avaliação de linhagens de outras regiões podem constituir o método de melhoramento mais simples, econômico e rápido para se obterem linhagens mais adaptadas ao cultivo (ALLARD, 1960). A adoção de linhagens adaptadas e recomendadas para uma região pode aumentar as receitas dos agricultores sem au mentar custos.

O objetivo deste trabalho foi o de avaliar características fenológicas e agronômicas de linhagens de feijão-mungo provenientes do ASIAN VEGETABLE RESEARCH AND DEVELOPMENT CENTER (AVRDC), Taiwan, R. O. C., para as condições da região sul do Brasil.

\section{MATERIAL E MÉTODOS}

$\begin{array}{cc} & \text { Foram conduzidos três experimentos na } \\ \text { Estação Experimental da UFSC/CCA, em }\end{array}$
Florianópolis, SC. Segundo a classificação de Köppen, o clima local é do tipo cfb, mesotérmico úmido e sem estação seca definida. A precipitação pluvial anual é de $1530 \mathrm{~mm}$, com uma evapotranspiração de $1038 \mathrm{~mm}$. A temperatura média é de $23,8^{\circ} \mathrm{C}$ no verão e de $16,8^{\circ} \mathrm{C}$ no inverno. $\mathrm{O}$ solo, caracterizado como Areia Quartzosa Hidromórfica distrófica, apresentou um $\mathrm{pH}$ em água de 4,4, teor de matéria orgânica (localizada somente na camada superficial) variando entre 4 e $5 \%$, níveis de fósforo de $1,0 \mathrm{Cmo} \ell \mathrm{c} \cdot \mathrm{dm}^{-1}$ e de potássio entre 20 e $30 \mathrm{Cmo} \ell \mathrm{c} \cdot \mathrm{dm}^{-1}$.

As datas de semeadura foram as seguintes: 21/09/92 (primeiro), 24/09/93 (segundo) e 22/09/94 (terceiro). No primeiro experimento, foram utilizadas 13 linhagens (Tabela 1). Estas 13 linhagens, mais sete, VC 4443 A, VC 4355 A, VC 3541B, NM 51, Pusa 9173, NM 92 e NM 94, foram incluídas no segundo experimento. Para a condução do terceiro experimento, foram incluídas as 11 linhagens mais promissoras do segundo experimento, mais as linhagens V 3726, V 3476, VC 1974 A, VC 2764A, VC 2523 A, VC 1000, VC 2750 A, VC 2754A e VC 2719A.

As linhagens foram testadas no delineamento de blocos ao acaso, com quatro repetições. Cada parcela foi constituída por 4 linhas de $6 \mathrm{~m}$ de comprimento, espaçadas de $50 \mathrm{~cm}$. Foram utilizadas 20 sementes por metro de sulco, com $85 \%$ de poder germinativo. A adubação foi feita de acordo com a recomendação de PARK (1978) para a cultura de feijão-mungo (N: P2O5 : $\mathrm{K} 2 \mathrm{O}=30$ : 60: $100 \mathrm{~kg} . \mathrm{ha}^{-1}$ ). Por ocasião da semeadura, fez-se uma adubação básica com $15 \mathrm{~kg} \cdot \mathrm{ha}^{-1}$ de $\mathrm{N}, 60 \mathrm{~kg} \cdot \mathrm{ha}^{-1}$ de $\mathrm{P}_{2} \mathrm{O}_{5}$ e $100 \mathrm{~kg} \cdot \mathrm{ha}^{-1}$ de $\mathrm{K}_{2} \mathrm{O}$, respectivamente nas formas de sulfato de amônio, superfosfato simples e cloreto de potássio. $\mathrm{Na}$ época do florescimento, foi realizada a adubação de cobertura com $15 \mathrm{~kg} \cdot \mathrm{ha}^{-1} \mathrm{de}$ $\mathrm{N}$, na forma de uréia. Duas capinas foram feitas para controlar as plantas daninhas.

Durante o estádio de desenvolvimento, foram observadas as datas de formação da primeira flor e do primeiro legume maduro em cada parcela. Considerou-se como "formação da primeira flor" e "maturação do primeiro legume" a existência em $50 \%$ das plantas das parcelas com, pelo menos, uma flor aberta e um legume seco, respectivamente.

A colheita foi manual e realizada em uma única operação entre 80 e 85 dias após a semeadura no primeiro e no segundo experimento. No terceiro experimento, a colheita foi feita em duas vezes, sendo a primeira efetuada entre 80 e 85 dias e a segunda, entre 95 e 100 dias após a semeadura. A estatura da planta foi obtida pela média de 10 plantas 
Tabela 1 - Número de dias entre semeadura e formação da primeira flor (NF) e entre semeadura e primeiro legume maduro (NL), estatura da planta (AP), em $\mathrm{cm}$, peso de mil sementes (P1000), em gramas, e rendimento de grãos (RG), em kg.ha ${ }^{-1}$, de linhagens de feijão-mungo, Florianópolis, SC. 2000.

\begin{tabular}{|c|c|c|c|c|c|}
\hline Linhagens & $\mathrm{NF}$ & NL & AP & P1000 & RG \\
\hline \multicolumn{6}{|c|}{ Primeiro Experimento } \\
\hline VC 2764 B & 48 & $76 \mathrm{bc}^{*}$ & 46 & $64,5 \mathrm{c}$ & $1286 \mathrm{a}$ \\
\hline VC $3890 \mathrm{~B}$ & 49 & $77 \quad \mathrm{c}$ & 40 & $63,8 \quad \mathrm{c}$ & $1260 \mathrm{a}$ \\
\hline Chun Nam 2 & 44 & $72 a b$ & 42 & 52,3 b & $1246 \mathrm{a}$ \\
\hline VC $4503 \mathrm{~A}$ & 45 & $73 \mathrm{ab}$ & 44 & $76,4 \quad \mathrm{~d}$ & $1209 a b$ \\
\hline Chun Nam 5 & 46 & $74 \mathrm{~b}$ & 44 & 52,2 b & $1157 \mathrm{ab}$ \\
\hline VC $4386 \mathrm{~A}$ & 48 & $76 \mathrm{bc}$ & 50 & $74,3 \quad \mathrm{~d}$ & $1106 \mathrm{ab}$ \\
\hline VC 1178 B & 47 & $75 \mathrm{bc}$ & 46 & 75,5 & $1089 \mathrm{ab}$ \\
\hline VC 1973 A & 47 & $75 \mathrm{bc}$ & 49 & 74,4 & $1073 \mathrm{ab}$ \\
\hline VC1973 C & 47 & $75 \mathrm{bc}$ & 47 & $75,9 \quad \mathrm{~d}$ & $1067 \mathrm{ab}$ \\
\hline Chun Nam 4 & 43 & $71 \mathrm{a}$ & 40 & 53,0 b & $1046 \mathrm{ab}$ \\
\hline VC 4152 B & 45 & $73 \mathrm{ab}$ & 41 & $72,7 \mathrm{~cd}$ & $984 \mathrm{ab}$ \\
\hline ML 267 & 45 & $73 \mathrm{ab}$ & 45 & $33,8 \mathrm{ab}$ & $803 \mathrm{~b}$ \\
\hline VC $2768 \mathrm{~A}$ & 49 & $77 \mathrm{c}$ & 47 & $72,0 \quad \mathrm{~cd}$ & $782 \mathrm{~b}$ \\
\hline Média & 46 & 74 & 45 & 65 & 1085 \\
\hline $\mathrm{CV} \%$ & 4,10 & 2,54 & 7,27 & 20,38 & 14,61 \\
\hline \multicolumn{6}{|c|}{ Segundo Experimento } \\
\hline VC 3890 B & $49 \mathrm{a}$ & $77 \mathrm{a}$ & $31,3 \mathrm{ab}$ & 62,2 bc & $1422 \mathrm{a}$ \\
\hline VC $4503 \mathrm{~A}$ & $45 \mathrm{~b}$ & $73 \mathrm{c}$ & $30,0 \mathrm{ab}$ & 76,4 a & $1323 \mathrm{a}$ \\
\hline VC $2764 \mathrm{~B}$ & $48 \mathrm{a}$ & $76 \mathrm{a}$ & $38,2 \mathrm{a}$ & $64,5 \mathrm{~b}$ & $1296 \mathrm{a}$ \\
\hline Chun Nam 2 & $45 \mathrm{~b}$ & $73 \mathrm{~b}$ & $31,0 \mathrm{ab}$ & 52,9 cd & $1266 \mathrm{a}$ \\
\hline VC 4443 A & $49 \mathrm{a}$ & $77 \mathrm{a}$ & $30,4 \mathrm{ab}$ & $61,2 \mathrm{bc}$ & $1177 \mathrm{ab}$ \\
\hline Chun Nam 5 & $45 \mathrm{~b}$ & $73 \mathrm{~b}$ & $32,5 \mathrm{a}$ & $52,7 \mathrm{~cd}$ & $1168 \mathrm{ab}$ \\
\hline VC $4386 \mathrm{~A}$ & $49 a$ & $77 \mathrm{a}$ & $35,3 \mathrm{a}$ & 74,9 a & $1125 \mathrm{ab}$ \\
\hline VC 1178B & $46 \mathrm{~b}$ & $74 \mathrm{~b}$ & $32,4 \mathrm{ab}$ & $75,9 \mathrm{a}$ & $1095 \mathrm{ab}$ \\
\hline VC 1973 A & $47 \mathrm{ab}$ & $75 \mathrm{ab}$ & $29,4 \mathrm{ab}$ & $74,4 \mathrm{a}$ & $1088 \mathrm{ab}$ \\
\hline VC 1973C & $46 \mathrm{~b}$ & $74 \mathrm{~b}$ & $30,0 \mathrm{ab}$ & $76,0 \mathrm{a}$ & $1079 \mathrm{ab}$ \\
\hline Chun Nam 4 & $42 \mathrm{c}$ & $70 \mathrm{c}$ & $27,4 \quad b$ & $53,3 \quad \mathrm{c}$ & $1056 \mathrm{ab}$ \\
\hline VC $4355 \mathrm{~A}$ & $49 a$ & $77 \mathrm{a}$ & $25,6 \mathrm{~b}$ & $62,6 \mathrm{bc}$ & $1016 \mathrm{ab}$ \\
\hline VC $4152 \mathrm{~B}$ & $44 \mathrm{bc}$ & $72 \mathrm{bc}$ & $29,9 \mathrm{ab}$ & $72,0 \mathrm{ab}$ & $964 \mathrm{ab}$ \\
\hline VC $3541 \mathrm{~B}$ & $49 \mathrm{a}$ & $77 \mathrm{a}$ & $30,6 \mathrm{ab}$ & $64,8 \mathrm{~b}$ & $867 a b$ \\
\hline ML 267 & $44 \mathrm{bc}$ & $72 \mathrm{bc}$ & $30,6 \mathrm{ab}$ & 33,0 & $823 \mathrm{ab}$ \\
\hline VC $2768 \mathrm{~A}$ & $48 \mathrm{a}$ & $76 \mathrm{a}$ & $27,0 \mathrm{~b}$ & $72,5 \mathrm{ab}$ & $802 a b$ \\
\hline NM 51 & $45 \mathrm{~b}$ & $73 \mathrm{~b}$ & $29,0 \mathrm{ab}$ & 41,3 & $761 \mathrm{ab}$ \\
\hline Pusa 9173 & $43 \mathrm{c}$ & $71 \mathrm{c}$ & $27,0 \quad b$ & $45,4 \quad \mathrm{~d}$ & $732 a b$ \\
\hline NM 92 & $42 \mathrm{c}$ & $70 \mathrm{c}$ & $23,1 \mathrm{~b}$ & $69,0 \mathrm{ab}$ & $625 \mathrm{~b}$ \\
\hline NM 94 & $44 \mathrm{bc}$ & $72 b c$ & $22,5 \mathrm{~b}$ & $63,8 \mathrm{bc}$ & $619 \mathrm{~b}$ \\
\hline Média & 46 & 74 & 29,7 & 62,4 & 1015 \\
\hline $\mathrm{CV} \%$ & 5,26 & 3,27 & 12,42 & 20,06 & 23,07 \\
\hline \multicolumn{6}{|c|}{ Terceiro Experimento } \\
\hline VC3890 B & $41 \mathrm{~b}$ & $78 \mathrm{~b}$ & $61,0 \mathrm{bcd}$ & & $1176 \mathrm{a}$ \\
\hline VC 1973 C & $42 \mathrm{~b}$ & $78 \mathrm{~b}$ & 59,7 abcd & & $1153 \mathrm{a}$ \\
\hline VC 2764 B & $42 \mathrm{~b}$ & $78 \mathrm{~b}$ & $45,3 \mathrm{ab}$ & & $1145 \mathrm{a}$ \\
\hline VC $4443 \mathrm{~A}$ & $41 \mathrm{~b}$ & $78 \mathrm{~b}$ & $57,0 \mathrm{abcd}$ & & $1091 \mathrm{ab}$ \\
\hline V 3726 & $42 \mathrm{~b}$ & $78 \mathrm{~b}$ & 51,6 abcd & & $1053 \mathrm{abc}$ \\
\hline VC 1973 A & $40 \mathrm{ab}$ & $77 \mathrm{~b}$ & $55,2 \mathrm{abcd}$ & & 1014 abcd \\
\hline V 3476 & $38 \mathrm{ab}$ & $75 \mathrm{ab}$ & $48,6 \mathrm{abc}$ & & 940 abcde \\
\hline VC $3541 \mathrm{~B}$ & $40 \mathrm{ab}$ & $77 \mathrm{~b}$ & $63,8 \mathrm{~d}$ & & 830 abcdef \\
\hline VC 1178 B & $38 \mathrm{ab}$ & $73 \mathrm{ab}$ & 57,7 abcd & & 791 abcdefg \\
\hline VC 1974 A & $40 \mathrm{ab}$ & $77 \mathrm{~b}$ & $63,0 \mathrm{~cd}$ & & 778 abcdefg \\
\hline VC $4503 \mathrm{~A}$ & $36 a b$ & $73 \mathrm{ab}$ & $44,5 \mathrm{a}$ & & 709 bcdefg \\
\hline VC 2764 A & $40 \mathrm{ab}$ & $78 \mathrm{~b}$ & $61,8 \mathrm{~cd}$ & & 662 cdefg \\
\hline VC $4152 \mathrm{~B}$ & $33 \mathrm{a}$ & $70 \mathrm{a}$ & $60,7 \mathrm{bcd}$ & & 582 \\
\hline VC $2523 \mathrm{~A}$ & $39 \mathrm{ab}$ & $75 \mathrm{ab}$ & $60,8 \mathrm{bcd}$ & & 571 \\
\hline VC 1000 & $36 \mathrm{ab}$ & $73 \mathrm{ab}$ & 56,5 abcd & & 531 \\
\hline VC $2768 \mathrm{~A}$ & $36 \mathrm{ab}$ & $70 \mathrm{a}$ & 54,9 abcd & & 526 \\
\hline $\mathrm{VC} 4386 \mathrm{~A}$ & $39 \mathrm{ab}$ & $76 \mathrm{~b}$ & 58,0 abcd & & 508 \\
\hline VC $2750 \mathrm{~A}$ & $41 \mathrm{~b}$ & $78 \mathrm{~b}$ & 47,6 abc & & 502 \\
\hline VC $2754 \mathrm{~A}$ & $38 \mathrm{ab}$ & $75 \mathrm{ab}$ & 58,3 abcd & & 426 \\
\hline VC $2719 \mathrm{~A}$ & $33 \mathrm{a}$ & $70 \mathrm{a}$ & 53,5 abcd & & 366 \\
\hline Média & 39 & 75 & 56,0 & & 768 \\
\hline CV\% & 7,10 & 3,86 & 10,37 & & 34,8 \\
\hline
\end{tabular}

*Médias não seguidas da mesma letra, na vertical e dentro de cada experimento diferiram entre si pelo teste de Tukey em nível de $5 \%$ de probabilidade de uso.

tomadas ao acaso nas parcelas, por ocasião da colheita para todos os experimentos. Foi medido o comprimento entre o colo da planta e o seu meristema apical. As plantas das duas linhas centrais de cada parcela foram colhidas para a determinação do rendimento e o peso de mil sementes. $O$ peso de mil sementes e o rendimento foram corrigidos para $12 \%$ do teor de água da semente.

Todas as determinações descritas anteriormente foram submetidas à análise de variância, utilizando-se o F-teste com a finalidade de testar as diferenças entre os tratamentos. Para a comparação de médias, adotou-se o teste de Tukey em nível de $5 \%$ de probabilidade de uso. Para avaliar o grau de relação entre as variáveis, realizou-se uma analise de correlação.

\section{RESULTADOS E DISCUSSÃO}

Primeiro experimento: As variáveis, formação da primeira flor e estaturas das plantas não apresentaram diferença significativa entre os genótipos, enquanto para primeiro legume maduro, peso de mil sementes e rendimento de grão, houve diferença significativa entre os genótipos. As linhagens mais produtivas foram: VC 2764B, VC 3890B e Chun Nam 2 , com rendimento superior a $1240 \mathrm{~kg} \cdot \mathrm{ha}^{-1} \mathrm{e}$ as menos produtivas foram: ML 267 e VC 2768A (Tabela 1).

A formação da primeira flor correlacionou-se de forma positiva e significativa com o primeiro legume maduro, com a estatura das plantas e com o peso de mil sementes (Tabela 2). As linhagens VC 4386 A, VC 1178B, VC 1973 A, VC 1973 C e VC 2768A apresentaram o primeiro legume maduro mais tardiamente, com valores de altura de plantas e peso de mil sementes maiores que as médias das linhagens avaliadas. As linhagens mais precoces para a maturação do primeiro legume foram Chun Nam 2, Chun Nam 5, Chun Nam 4 e ML 267, com menores valores de estatura de plantas e de peso de mil sementes (Tabela 1).

Segundo experimento: Houve correlação positiva e significativa entre formação da primeira flor e primeiro legume maduro e ambas correlacionaram-se positivamente com a estatura das plantas, o peso de mil sementes e a produtividade (Tabela 2), ou seja, a tendência foi de quanto mais precoce a linhagem, menor a sua estatura, menor o peso de mil sementes e menor a produtividade. 
Tabela 2 - Coeficientes de correlação entre as variáveis número de dias entre semeadura e formação da primeira flor (NF) e entre semeadura e primeiro legume maduro (NL), estatura da planta (AP), peso de mil sementes (P1000) e rendimento de grãos (RG) de linhagens de feijão-mungo. Florianópolis, SC. 2000.

\begin{tabular}{|c|c|c|c|c|}
\hline & NL & $\mathrm{AP}$ & $\mathrm{P} 1000$ & $\mathrm{RG}$ \\
\hline \multicolumn{5}{|c|}{ Primeiro Experimento } \\
\hline $\begin{array}{l}\text { NF } \\
\text { NL } \\
\text { AP } \\
\text { P1000 }\end{array}$ & $0,99 *$ & $\begin{array}{l}0,51 * \\
0,51 *\end{array}$ & $\begin{array}{l}0,51 * \\
0,51 * \\
0,35 *\end{array}$ & $\begin{array}{c}0,02 \mathrm{NS} \\
0,02 \mathrm{NS} \\
-0,22 \mathrm{NS} \\
0,21 \mathrm{NS}\end{array}$ \\
\hline \multicolumn{5}{|c|}{ Segundo Experimento } \\
\hline $\begin{array}{l}\text { NF } \\
\text { NL } \\
\text { AP } \\
\text { P1000 }\end{array}$ & $0,99 *$ & $\begin{array}{l}0,46^{*} \\
0,46^{*}\end{array}$ & $\begin{array}{c}0,35^{*} \\
0,35^{*} \\
0,08 \mathrm{NS}\end{array}$ & $\begin{array}{l}0,45^{*} \\
0,45^{*} \\
0,69^{*} \\
0,26^{*}\end{array}$ \\
\hline \multicolumn{5}{|c|}{ Terceiro Experimento } \\
\hline $\begin{array}{l}\text { NF } \\
\text { NL } \\
\text { AP } \\
\text { P1000 }\end{array}$ & $0,95^{*}$ & $\begin{array}{l}0,02 \mathrm{NS} \\
0,06 \mathrm{NS}\end{array}$ & $\begin{array}{c}0,36^{*} \\
0,36^{*} \\
-0,02 \mathrm{NS}\end{array}$ & $\begin{array}{c}0,69^{*} \\
0,63^{*} \\
-0,07 \mathrm{NS} \\
0,73^{*}\end{array}$ \\
\hline
\end{tabular}

NS = Não significativo;

* = significativo em nível de $5 \%$

As estaturas das plantas no segundo experimento foram inferiores às observadas no primeiro experimento (Tabela 1). Esta característica da planta foi a que mais se correlacionou com a produtividade (Tabela 2). Isso ocorreu provavelmente, porque as plantas de baixa estatura tendem a produzir menor número de flores/planta e conseqüentemente, menor número de legumes/planta ou ainda, menor número de sementes/legume.

A linhagem VC 3890B, originária das Filipinas, foi a que apresentou o maior rendimento, com 1422kg.ha ${ }^{-1}$, seguida da VC $4503 \mathrm{~A}$, de Taiwan, foi a segunda em rendimento, com $1323 \mathrm{~kg} \mathrm{ha}^{-1} \mathrm{e}$ da linhagem VC 2764B, das Filipinas, foi a terceira em rendimento, de $1296 \mathrm{~kg} . \mathrm{ha}^{-1}$. A linhagem Chun Nam 2, originária da Coréia, também se destacou com rendimento acima de $1200 \mathrm{~kg} \cdot \mathrm{ha}^{-1}$. As linhagens NM 92 e NM 94, originárias do Paquistão, apresentaram os piores rendimentos, com $625 \mathrm{~kg} . \mathrm{ha}^{-1}$ e $619 \mathrm{~kg} . \mathrm{ha}^{-1}$, respectivamente (Tabela 1 ).

Terceiro experimento: As linhagens mais promissoras do primeiro e do segundo experimento foram reavaliadas no terceiro experimento junto com mais nove linhagens novas, introduzidas do AVRDC, Taiwan. O período para a formação da primeira flor nesse ensaio, variou entre 33 e 42 dias após o plantio. Esse período foi um pouco menor que os resultados apresentados no primeiro e no segundo experimento (Tabela 1), mas ainda foi maior que o constado por DUQUE $\boldsymbol{e t}$ al.
(1987). Esses autores, em um experimento com 21 linhagens de feijão-mungo, em Itaguaí, RJ, encontraram valores de 30 e 35 dias após a semeadura para o início do florescimento destas linhagens. A variação do período do início do florescimento foi devido, principalmente, às diferenças climáticas entre os anos e entre os dois locais de plantio, pois os presentes experimentos foram realizados em Florianópolis, SC, que apresenta uma latitude maior e portanto menores temperaturas do que as encontradas em Itaquaí, RJ. Nesta condição, as linhagens aqui testadas levaram mais tempo para completar a soma térmica necessária para a entrada no período de florescimento.

Houve correlação positiva e significativa entre a formação da primeira flor e o primeiro legume maduro no segundo e terceiro experimento e ambas correlacionaram-se positivamente com o peso de mil sementes e a produtividade (Tabela 2), ou seja, houve uma tendência de quanto mais precoce a linhagem, menor o peso de mil sementes e menor a produtividade. Isto, provavelmente, ocorreu em consequiência das linhagens precoces ainda não terem o seu arcabouço vegetativo totalmente formado por ocasião do florescimento, de maneira que a produção e a translocação de fotossimilados para o desenvolvimento dos grãos foi menor do que nas linhagens que floresceram um pouco mais tarde, o que se refletiu na menor produção.

As linhagens VC 2719 A, VC 2754 A, VC 4152B, VC 3541B e V 3476 apresentaram maior uniformidade de maturação, com mais de $90 \%$ das vagens colhidas na primeira colheita e as VC 2523A e VC 2750A apresentaram menor uniformidade, com menos de $60 \%$ das vagens colhidas na primeira colheita. A média do terceiro experimento mostrou que $78 \%$ dos legumes foram colhidos na primeira colheita, sendo que os restantes $22 \%$ foram colhidos 15 dias após a primeira colheita.

A colheita do feijão-mungo é laboriosa e, geralmente, são necessárias de duas a quatro colheitas de legumes maduros a intervalos de 7 a 15 dias (ASIAN, 1976), dependendo das condições climáticas. Em Itaguaí, RJ, duas colheitas, feitas entre os 63 e 69 dias após o plantio, foram suficientes para a maioria das variedades estudadas. No entanto, algumas variedades possibilitaram, mais duas colheitas (81 e 95 dias), as quais, representaram menos que $10 \%$ do total das duas primeiras colheitas (DUQUE et al., 1987). Na Austrália, uma colheita foi suficiente, quando a maturação dos legumes coincidiu com temperaturas amenas, dias curtos e período de baixa precipitação. Estas condições, normalmente, ocorrem no plantio de verão, quando a colheita é feita no outono (LAWN et al., 1988). 
O primeiro e segundo experimentos, realizados no mesmo local, registraram um rendimento médio de $1085 \mathrm{~kg} \cdot \mathrm{ha}^{-1}$ e $1015 \mathrm{~kg} \cdot \mathrm{ha}^{-1}$, respectivamente. A média obtida no terceiro experimento foi baixa devido, principalmente, à intensidade da chuva durante o estádio de enchimento de grãos, que causou uma redução no peso de mil sementes, e, conseqüentemente, a redução do rendimento (Tabela 1 ).

No primeiro e segundo experimentos, realizados no mesmo local nos anos anteriores, as linhagens mais produtivas foram: VC 3890B, VC 2764B e VC 4503A, com rendimentos acima de $1200 \mathrm{~kg} \cdot \mathrm{ha}^{-1}$. No terceiro experimento, as linhagens VC 3890B e VC 2764B repetiram o bom desempenho, ficando entre as mais produtivas; entretanto, a linhagem VC 4503A apresentou produção intermediária, com rendimento de 709kg.há1. A linhagem VC 4443A que, no segundo experimento alcançou um bom rendimento, com $1177 \mathrm{~kg} \cdot \mathrm{ha}^{-1}$, no terceiro experimento repetiu este bom desempenho,com 1091 kg.ha ${ }^{-1}$ (Tabela 1).

As médias dos rendimentos dos três experimentos foram de 1085,1015 e $768 \mathrm{~kg} . \mathrm{ha}^{-1}$, respectivamente, valores inferiores à média obtida por DUQUE et al. (1987), em Itaguaí, RJ, que foi de $1542 \mathrm{~kg} \cdot \mathrm{ha}^{-1}$. As linhagens V 3476 e VC 1000 testadas por DUQUE et al. (1987), em Itaguaí, RJ, apresentaram ótimos rendimentos, estando entre as mais produtivas $\left(1796\right.$ e 1766kg.ha ${ }^{-1}$, respectivamente), mas, neste terceiro experimento, não repetiram aquele bom rendimento, apresentando produções intermediárias com 940 e $531 \mathrm{~kg} \cdot \mathrm{ha}^{-1}$, respectivamente. Os dois plantios foram realizados na época da "seca" e esta diferença se deve às diferenças de temperatura e dos solos, principalmente, às diferenças dos solos dos dois experimentos, sendo que este foi realizado em um solo muito arenoso, de $\mathrm{pH}$ baixo e teores de fósforo baixíssimos, enquanto em Itaguaí, RJ, o experimento foi realizado em solo Podzólico, com um $\mathrm{pH}$ mais alto e maiores teores de fósforo, o que propiciou uma condição edáfica mais favorável a um maior desenvolvimento das plantas.

A linhagem VC 2719A, neste experimento, apresentou o pior rendimento. Em outro experimento conduzido por SAYÃO et $\boldsymbol{a l}$. (1991), em Itaguaí, RJ, esta linhagem também apresentou uma baixa produção e valores baixos para os componentes de rendimento, demonstrando não ser uma boa linhagem para as nossas condições.

Todas as linhagens testadas nos três experimentos, apresentaram porte ereto, legumes de coloração preta, quando maduros, e sementes de coloração verde. Não se observou acamamento em nenhum das linhagens. Também não se observou ataques de pragas e/ou doenças que pudessem causar prejuízos significativos ao rendimento das plantas. Algumas linhagens foram levemente atacadas pelo míldio pulverulento (Erysiphe polygoni) e pela mancha-de cercospora (Cercospora sp.).

Em caso de plantios extensos, o feijãomungo apresenta a possibilidade de colheita mecânica, método já utilizado na Austrália (LAWN et al., 1988). Além disso, esta cultura ocupa o solo por pouco tempo e sem problemas em relação a pragas e doenças. Neste trabalho, as melhores linhagens apresentaram uma boa produtividade, variando de 1145 a $1422 \mathrm{~kg} . \mathrm{ha}^{-1}$, quando comparada à produtividade do feijão comum no Brasil, que foi de 559kg.ha- ${ }^{-1}$ IBGE (2000). O feijão-mungo pode, também, ser utilizado como uma boa opção para forragem, feno, adubo verde e cultura de cobertura (VIEIRA, 1992), o que demonstra a possibilidade de se tornar uma cultura de interesse para os pequenos agricultores do Sul do País.

\section{CONCLUSÕES}

As linhagens de feijão-mungo apresentaram diferenças significativas de ciclo biológico, estatura de plantas, peso de mil sementes e rendimento. As linhagens mais tardias foram, em geral, as mais produtivas, com destaque para VC 3890B, VC 1973C e VC 2764B.

A maioria das linhagens ainda não matura os legumes uniformemente.

\section{REFERÊNCIAS BIBLIOGRÁFICAS}

ALLARD, R.W. Principles of plant breeding. New York: J.Wiley \& Sons, 1960. 485p.

ASIAN VEGETABLE RESEARCH AND DEVELOPMENT CENTER (Tainan, Taiwan). Mungbean report, Shanhua, Taiwan, 1976. v.75. 72p

BARRADAS, C.A.A., SAYÃO, F.A.D., DUQUE, F.F. Feijãomungo:uma alternativa protéica na alimentação. Rio de Janeiro : EMBRAPA-CNPBS, 1989. 4p. (EMBRAPA/ CNPBS. Comunicado Técnico, 4. )

DUQUE, F.F., PESSANHA, G.G. Comportamento de 10 cultivares de mungo verde nos períodos das águas e das secas em condições de campo. Pesq Agropec Brasília, v.25, p.963969,1990

DUQUE, F.F., PESSANHA, G.G., QUEIROZ,P.H. de S. Estudo preliminar sobre o comportamento de 21 cultivares de feijãomungo em Itaguaí-RJ. Pesq Agropec Brasília, v.22, p.593598, 1987.

IBGE. Levantamento sistemático da produção agrícola. Rio de Janeiro : IBGE, Deagro, dez 1999 - abr/mai 2000. 
LAWN, R.J., CHAY, P.M., IMRIE, B.C. The mungbean industry in Australia. In: INTERNATIONAL MUNGBEAN SYMPOSIUM, 2, 1987, Bangkok, Thailand. Proceedings... Tainan, Taiwan : AVRDC, 1988. p.560-569.

PARK, H.G. Sugested cultural practices for mungbean. Tainan, Taiwan : AVRDC, 1978. 4p.

SANGAKKARA, U.R., SOMARATNE, H.M. Sources, storage condition and quality of mungbean seeds cultivation in Sri Lanka. Seed Science \& Technology, v.16, p.5-10, 1988.

SAYÃO, F.A.D., BRIOSO, R.S.T., DUQUE, F.F. Comportamento de linhagens de mungo verde em condições de campo em Itaguaí, RJ. Pesq Agropec, Brasília, v.26, p.659-664, 1991.
TSOU, S.C.S., HSU, M.S. The potential roles of mungbean as a diet component in Asia. In: R.COWELL, (ed.) THE FIRST INTERNATIONAL MUNGBEAN SYMPOSIUM. Proceedings.... Tainan, Taiwan : AVRDC, 1978. p.40-45.

VIEIRA, R.F. Comparação de feijões dos gêneros Vigna e Phaseolus com o feijão comum (Phaseolus vulgaris L.). Viçosa, 1989. 213p. Tese (Doutorado) - Curso de Pósgraduação, UFV, 1989.

VIEIRA, R.F. Cultura do feijão-mungo. Informe Agropecuário,v.16, n.174, p.37-46, 1992. 